\begin{tabular}{|c|l|}
\hline Title & Linear A nalysis of Dispersive Waves in Bubbly Flows Based on A veraged Equations \\
\hline Author(s) & Y ano, Takeru; Egashira, Ryu; Fujikawa, Shigeo \\
\hline Citation & Journal of the Physical Society of Japan, 75(10), 104401 \\
\hline https://doi.org/10.1143/PSJ.75.104401 \\
\hline Issue Date & 2006-10 \\
\hline Doc URL & http://hdl.handle.net/2115/16871 \\
\hline Rights & Copyright $\odot$ 2006 The Physical Society of Japan \\
\hline Rights(URL) & http:/\$psj.jpap.jp/ink?JPSJ/75/104401// \\
\hline Type & article (author version) \\
\hline Information & \\
\hline
\end{tabular}

Instructions for use 


\title{
Linear Analysis of Dispersive Waves in Bubbly Flows Based on Averaged Equations
}

\author{
Takeru Yano*, Ryu Egashira ${ }^{1}$ and Shigeo Fujikawa \\ Division of Mechanical and Space Engineering, Hokkaido University, Sapporo 060-8628, Japan \\ ${ }^{1}$ Sugino Machine Limited, Uozu 937-8511, Japan \\ (Received August 14, 2006)
}

\begin{abstract}
One-dimensional linear dispersive waves in water flows containing a number of small spherical air bubbles are analytically studied on the basis of a set of averaged equations recently derived by the present authors. The set of equations consists of the conservation laws for gas and liquid phases and the equation of motion of bubble wall. In addition to the volumeaveraged pressure in each phase, the surface-averaged liquid pressure at the bubble wall is incorporated. The compressibility of water is taken into account as well as that of gas in bubbles, and a model of virtual mass force is included, although the Reynolds stress, viscosity, heat conductivity, and the phase change across the bubble wall are disregarded. The results are summarized as follows: (i) the waves are decomposed into the fast mode, slow mode, and convection mode (void wave). (ii) In the uniform flows, the three modes stably exist for all real wave numbers. (iii) In the limit of infinitesimal void fraction, the explicit representation of the elementary solution is obtained. (iv) The instability does not appear in the range where the present averaged equations are applicable.
\end{abstract}

KEYWORDS: linear dispersive wave, averaged equations, bubbly flow, bubble dynamics, void wave

\section{Introduction}

The characteristics of wave motions in bubbly liquids are considerably different from those in a pure (single phase) fluid, as shown by van Wijngaarden, ${ }^{1)}$ Caflisch et al., ${ }^{2)}$ Pauchon and Banerjee, ${ }^{3,4)}$ Commander and Prosperetti, ${ }^{5)}$ Lahey, ${ }^{6)}$ Park et al., ${ }^{7)}$ Gavrilyuk and Saurel, ${ }^{8)}$ and many other researchers. The appearance of dispersion is common there. The considerable decrease of the speed of sound of long wavelength is well known as one of the most remarkable features of the waves in the bubbly liquids. ${ }^{1}$ ) The propagation of the so-called void wave (or convection wave) and the onset of its instability have also been major topics. ${ }^{6)}$ The dynamics of bubble oscillation, however, has not always been included into the analyses. In this paper, we shall analytically study the linear wave motions in a bubbly flow, where a number of small spherical gas bubbles are contained in a compressible fluid (water), and the bubble oscillations can be excited by the wave motions.

\footnotetext{
*E-mail address: yano@mech-me.eng.hokudai.ac.jp
} 
Recently, the present authors have analyzed linear wave motions in a bubbly liquid uniform and at rest on the basis of a set of averaged equations derived by ourselves. ${ }^{9}$ The set of equations consists of mass and momentum conservation laws for each phase and the Keller equation of motion of bubble wall. ${ }^{10)}$ According to Jones and Prosperetti, ${ }^{11)}$ the surfaceaveraged liquid pressure at the bubble wall is introduced as a dependent variable as well as the volume-averaged liquid and gas pressures. The compressibility of fluid in the liquid phase is taken into account, Focusing on the fundamental property in wave propagation processes, the Reynolds stress, viscosity, and heat conductivity, which are mainly responsible for wave attenuation, are neglected. The phase change across the bubble wall are not considered for simplicity. On the basis of these equations, we have studied the one-dimensional linear wave motions for the case that the bubbly liquid is uniform and at rest. ${ }^{9)}$ We have demonstrated that a fast mode, which is induced by the compressibility of fluid in the liquid phase, appears and sometimes behaves like a precursor of the classical pressure wave (slow mode). In the following, we shall extend the previous study to the linear wave motions in steady bubbly flows.

\section{Governing Equations}

We shall analyze the bubbly water flows containing a number of small spherical air bubbles on the basis of the averaged equations. The system of governing equations is composed of the mass and momentum conservation laws for gas and liquid phases, the Keller equation for the oscillation of spherical bubble, the equations of state for gas and liquid, and so on. ${ }^{9)}$ For the one-dimensional flows the mass and momentum conservation laws can be written as follows (see also the book by Drew and Passman ${ }^{12)}$ ):

$$
\begin{gathered}
\frac{\partial}{\partial t}\left(\alpha \rho_{G}\right)+\frac{\partial}{\partial x}\left(\alpha \rho_{G} u_{G}\right)=0, \\
\frac{\partial}{\partial t}\left[(1-\alpha) \rho_{L}\right]+\frac{\partial}{\partial x}\left[(1-\alpha) \rho_{L} u_{L}\right]=0 \\
\frac{\partial}{\partial t}\left(\alpha \rho_{G} u_{G}\right)+\frac{\partial}{\partial x}\left(\alpha \rho_{G} u_{G}^{2}\right)+\alpha \frac{\partial p_{G}}{\partial x}=F, \\
\frac{\partial}{\partial t}\left[(1-\alpha) \rho_{L} u_{L}\right]+\frac{\partial}{\partial x}\left[(1-\alpha) \rho_{L} u_{L}^{2}\right] \\
+(1-\alpha) \frac{\partial p_{L}}{\partial x}+P \frac{\partial \alpha}{\partial x}=-F,
\end{gathered}
$$

where $\alpha$ is the volume fraction of gas phase $(0<\alpha<1), \rho$ is the density, $u$ is the velocity, $p$ is the pressure, $F$ represents the interfacial momentum transport, and the subscripts $G$ and $L$ denote the volume-averaged quantities in gas and liquid phases, respectively. In addition to the volume-averaged pressures $p_{G}$ and $p_{L}$, the liquid pressure averaged on the gas-liquid interface, $P$, is introduced in the system of equations. ${ }^{11)}$ The energy conservation laws are not considered, as consistent with the implicit assumption of constant temperature. 
In the previous paper, ${ }^{9)}$ as the interfacial momentum transport $F$, we have employed a model of virtual mass force,

$$
F=-\beta\left[\frac{D_{G}}{D t}\left(\alpha \rho_{L} u_{G}\right)-\frac{D_{L}}{D t}\left(\alpha \rho_{L} u_{L}\right)\right]
$$

with $\beta=1 / 2$ used by Kameda and Matsumoto. ${ }^{13)}$ However, the operator $u_{G}\left(D_{G} / D t\right)-$ $u_{L}\left(D_{L} / D t\right)$ in eq. (5) is not invariant under the Galilean transformation, $\tau=t, \xi=x-V t$, and $v=u-V$. We therefore introduce a different model, ${ }^{14,15)}$

$$
\begin{aligned}
F=-\beta_{1} \alpha \rho_{L}\left(\frac{D_{G} u_{G}}{D t}-\frac{D_{L} u_{L}}{D t}\right) & \\
& -\beta_{2} \rho_{L}\left(u_{G}-u_{L}\right) \frac{D_{G} \alpha}{D t}-\beta_{3} \alpha\left(u_{G}-u_{L}\right) \frac{D_{G} \rho_{L}}{D t},
\end{aligned}
$$

which is invariant under the Galilean transformation. The values of coefficients $\beta_{1}, \beta_{2}$, and $\beta_{3}$ may be set as $1 / 2$. The importance of such invariance has been discussed in detail by Drew et $a l .{ }^{16)}$ The previous analysis ${ }^{9)}$ has been confined to the linear waves in quiescent bubbly liquids, and hence the results are not affected by the unfavorable character of eq. (5). The drag is not included in $F$, because we neglect the effects responsible for wave attenuation, as mentioned in Introduction.

The conservation laws of mass and momentum for the bubbly liquid, eqs. (1)-(4), are augmented by the Keller equation, ${ }^{10}$

$$
\begin{aligned}
\left(1-\frac{1}{c_{L 0}} \frac{D_{G} R}{D t}\right) R \frac{D_{G}^{2} R}{D t^{2}}+\frac{3}{2}\left(1-\frac{1}{3 c_{L 0}} \frac{D_{G} R}{D t}\right)\left(\frac{D_{G} R}{D t}\right)^{2} & \\
= & \left(1+\frac{1}{c_{L 0}} \frac{D_{G} R}{D t}\right) \frac{P}{\rho_{L 0}}+\frac{R}{\rho_{L 0} c_{L 0}} \frac{D_{G}}{D t}\left(p_{L}+P\right),
\end{aligned}
$$

( $R$ is the radius of bubble, $c_{L 0}$ and $\rho_{L 0}$ are, respectively, the speed of sound and density in an unperturbed water), the Tait equation of state for water,

$$
\left(p_{L}+B\right) \rho_{L 0}^{n}=\left(p_{L 0}+B\right) \rho_{L}^{n},
$$

( $p_{L 0}$ is the water pressure in the unperturbed state, $B=304.9 \mathrm{MPa}$, and $n=7.15$ ), the isothermal equation of state for gas,

$$
p_{G} \rho_{G 0}=p_{G 0} \rho_{G},
$$

$\left(\rho_{G 0}\right.$ and $p_{G 0}$ are, respectively, the density and pressure inside the bubble in the unperturbed state), the conservation law of mass for the gas inside each bubble,

$$
\rho_{G} R^{3}=\rho_{G 0} R_{0}^{3}
$$

( $R_{0}$ is the radius of the bubble in the unperturbed state), and the pressure balance at the interface,

$$
p_{G}-\left(p_{L}+P\right)=\frac{2 \sigma}{R}
$$


where $\sigma$ is the surface tension. Note that the variables in the initial undisturbed state, $\rho_{G 0}$, $p_{G 0}, \rho_{L 0}, p_{L 0}$, and $R_{0}$, are all constants.

All the variables are nondimensionalized and linearized as follows

$$
\begin{aligned}
& x=L x^{*}, \quad t=\frac{L}{c_{L 0}} t^{*}, \quad \frac{R}{R_{0}}=1+\epsilon R^{\prime}, \\
& \frac{u_{G}}{c_{L 0}}=u_{G 0}^{*}+\epsilon u_{G}^{\prime}, \quad \frac{u_{L}}{c_{L 0}}=u_{L 0}^{*}+\epsilon u_{L}^{\prime}, \\
& \alpha=\alpha_{0}^{*}\left(1+\epsilon \alpha^{\prime}\right), \quad \frac{p_{L}}{\rho_{L 0} c_{L 0}^{2}}=p_{L 0}^{*}\left(1+\epsilon p_{L}^{\prime}\right), \\
& \frac{p_{G}}{\rho_{L 0} c_{L 0}^{2}}=p_{G 0}^{*}\left(1+\epsilon p_{G}^{\prime}\right), \quad \frac{P}{\rho_{L 0} c_{L 0}^{2}}=\epsilon P^{\prime}, \\
& \frac{\rho_{G}}{\rho_{L 0}}=\rho_{G 0}^{*}\left(1+\epsilon \rho_{G}^{\prime}\right), \quad \frac{\rho_{L}}{\rho_{L 0}}=1+\epsilon \rho_{L}^{\prime},
\end{aligned}
$$

where $L$ is a characteristic length of the initial perturbation, $\epsilon(\ll 1)$ is a measure of the amplitude of the perturbation, the prime indicates the normalized perturbation of the order of unity. The quantities with $*$ are the nondimensionalized ones and the subscript 0 denotes the value in the unperturbed steady flow; $u_{G 0}^{*}, u_{L 0}^{*}, \alpha_{0}^{*}, p_{L 0}^{*}, p_{G 0}^{*}$, and $\rho_{G 0}^{*}$ are nondimensional constants, which are not necessarily of the order of unity. In the following analysis, only the nondimensional variables are used, and therefore the prime and the asterisk will be omitted.

As a result, we have the following set of the linearized equations: the mass conservation law in gas phase,

$$
\frac{\partial}{\partial t}(\alpha-3 R)+\frac{\partial}{\partial x}\left[u_{G 0}(\alpha-3 R)+u_{G}\right]=0
$$

the mass conservation law in liquid phase,

$$
\begin{gathered}
\frac{\partial}{\partial t}\left[\alpha_{0} \alpha+\left(\alpha_{0}-1\right) p_{L 0} p_{L}\right] \\
+\frac{\partial}{\partial x}\left[\alpha_{0} u_{L 0} \alpha+\left(\alpha_{0}-1\right)\left(p_{L 0} u_{L 0} p_{L}+u_{L}\right)\right]=0,
\end{gathered}
$$

the momentum conservation law in gas phase,

$$
\begin{gathered}
\frac{\partial}{\partial t}\left[\left(u_{G 0}-u_{L 0}\right)\left(\beta_{2} \alpha+\beta_{3} p_{L 0} p_{L}\right)+\left(\beta_{1}+\rho_{G 0}\right) u_{G}-\beta_{1} u_{L}\right] \\
+\frac{\partial}{\partial x}\left[u_{G 0}\left(u_{G 0}-u_{L 0}\right)\left(\beta_{2} \alpha+\beta_{3} p_{L 0} p_{L}\right)-3 p_{G 0} R\right. \\
\left.+\left(\beta_{1}+\rho_{G 0}\right) u_{G 0} u_{G}-\beta_{1} u_{L 0} u_{L}\right]=0,
\end{gathered}
$$

the momentum conservation law in liquid phase,

$$
\begin{gathered}
\frac{\partial}{\partial t}\left[\alpha_{0}\left(u_{G 0}-u_{L 0}\right)\left(\beta_{2} \alpha+\beta_{3} p_{L 0} p_{L}\right)\right. \\
\left.+\beta_{1} \alpha_{0}\left(u_{G}-u_{L}\right)+\left(\alpha_{0}-1\right) u_{L}\right] \\
+\frac{\partial}{\partial x}\left[\alpha_{0} u_{G 0}\left(u_{G 0}-u_{L 0}\right)\left(\beta_{2} \alpha+\beta_{3} p_{L 0} p_{L}\right)+\left(\alpha_{0}-1\right) p_{L 0} p_{L}\right.
\end{gathered}
$$




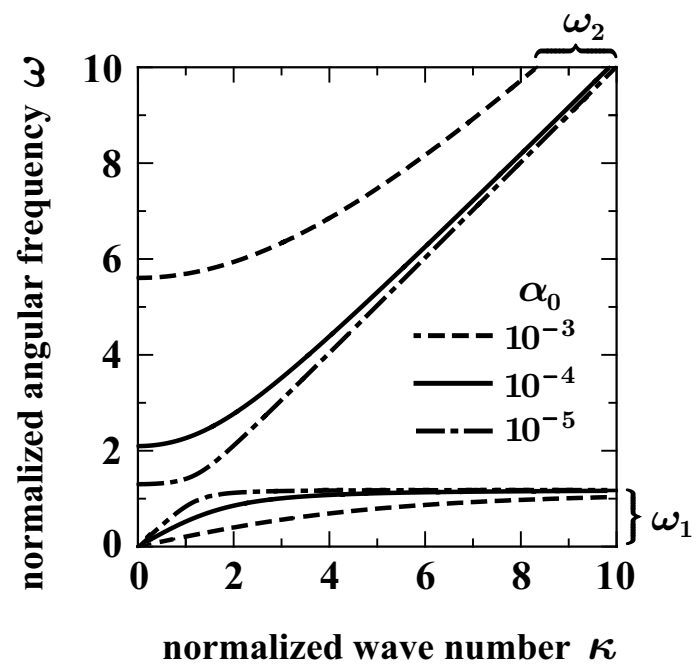

Fig. 1. The dispersion relation of fast and slow modes in the bubbly quiescent liquid: $u_{G 0}=u_{L 0}=$ $U=0, \delta=0.01$, and $R_{0}=1 \mathrm{~mm}$.

$$
\left.+\beta_{1} \alpha_{0}\left(u_{G 0} u_{G}-u_{L 0} u_{L}\right)+\left(\alpha_{0}-1\right) u_{L 0} u_{L}\right]=0,
$$

and the linearized Keller equation,

$$
\begin{gathered}
\frac{\partial R}{\partial t}+u_{G 0} \frac{\partial R}{\partial x}=S \\
\frac{\partial S}{\partial t}+u_{G 0} \frac{\partial S}{\partial x}=\frac{\left(2 \hat{\sigma}-3 p_{G 0}\right) R-p_{L 0} p_{L}}{\delta^{2}}
\end{gathered}
$$

where

$$
\delta=\frac{R_{0}}{L}, \quad \hat{\sigma}=\frac{\sigma}{\rho_{L 0} c_{L 0}^{2} R_{0}}, \quad p_{G 0}=p_{L 0}+2 \hat{\sigma} .
$$

In the equation of motion of bubble wall (18), we neglect some terms responsible for the wave attenuation due to acoustic radiation during the bubble oscillation.

\section{Linear Dispersive Waves}

The linear analysis is started by substituting a plane wave solution, i.e., $\alpha=$ $a \exp [i(\omega t-\kappa x)]$ etc., into the system of the linearized equations (13)-(18), where $a$ is a constant, $\kappa / L$ is a wavenumber ( $\kappa$ is a normalized wavenumber), and $\omega c_{L 0} / L$ is an angular frequency ( $\omega$ is a normalized angular frequency). The dispersion relation $f(\kappa, \omega)=0$ is obtained as the eigenequation (or secular equation) of the resulting system, where $f(\kappa, \omega)$ is a polynomial of sixth order in $\kappa$ and $\omega$. Its explicit representation is too long to be written here and we may proceed the discussion without showing it.

Here, we shall remark that the description on the basis of the averaged equations (1)-(11), and hence eqs. (13)-(18), may be subject to the restrictions,

$$
R_{0} \ll \sqrt[3]{V} \ll L \text { and } R_{0} \ll n_{0}^{-1 / 3} \ll \sqrt[3]{V}
$$


or

$$
\delta^{3} \ll \frac{V}{L^{3}} \ll 1 \text { and } L^{3} \delta^{3} n_{0} \ll 1 \ll V n_{0}
$$

where $V$ is the volume for averaging, $n_{0}$ is the number density of bubble in the unperturbed steady state, and $n_{0}{ }^{-1 / 3}$ represents a typical inter-bubble distance. The void fraction in the unperturbed state $\alpha_{0}$ therefore is sufficiently small compared with unity, i.e.,

$$
\alpha_{0}=\frac{4}{3} \pi L^{3} \delta^{3} n_{0} \ll 1 .
$$

The wavelength of the perturbation $\lambda=2 \pi L / \kappa$ has to be sufficiently large compared with $R_{0}$, and at least it is as large as $\sqrt[3]{V}$. This leads to another restriction,

$$
\frac{R_{0}}{\lambda}=\delta \kappa \ll 2 \pi
$$

\subsection{Waves in uniform bubbly flow}

Firstly, we shall consider the case that the gas and liquid phases move with the same speed in the same direction in the initial unperturbed state, namely $u_{G 0}=u_{L 0}=U$. Then, the dispersion relation can be written as

$$
\begin{gathered}
f(\kappa, \omega)=\left\{2 \beta_{1}\left[\kappa^{2}-(\omega-\kappa U)^{2}\right]\left[\delta^{2}(\omega-\kappa U)^{2}-\omega_{B}^{2}\right]\right. \\
+2 \alpha_{0} \beta_{1}(\omega-\kappa U)^{2}\left[3-2 \omega_{B}^{2}+2 \delta^{2}(\omega-\kappa U)^{2}\right] \\
-2 \alpha_{0}^{2} \beta_{1}(\omega-\kappa U)^{2}\left[3-\omega_{B}^{2}+\delta^{2}(\omega-\kappa U)^{2}\right] \\
-\alpha_{0} \kappa^{2}\left[\left(3+\beta_{1}\right) \omega_{B}^{2}-3\left(1+\beta_{1}\right) p_{L 0}+2 \beta_{1} \delta^{2}(\omega-\kappa U)^{2}\right] \\
\left.+3 \alpha_{0}^{2} \kappa^{2}\left(\omega_{B}^{2}-p_{L 0}\right)\right\}(\omega-\kappa U)^{2}=0,
\end{gathered}
$$

where $\omega_{B}=\sqrt{3 p_{L 0}+4 \hat{\sigma}}$ is the nondimensionalized eigenfrequency of a bubble in the initial unperturbed state. The linear dispersion relation is independent of the coefficients $\beta_{2}$ and $\beta_{3}$ in eq. (6). Note that we neglect $\rho_{G 0}$ here and hereafter since $\rho_{G 0}$ is small $\left(\cong 10^{-3}\right)$ and the result is not affected substantially.

In the case of $\beta_{1}=1 / 2$, for an arbitrary real $\kappa$ in all realistic conditions in bubbly flows of water and air bubbles, we have five real $\omega$ 's as the solutions of eq. (24),

$$
\begin{gathered}
\omega_{1 \pm}=\kappa U \pm \sqrt{\frac{A-\sqrt{A^{2}-4 B}}{2 \delta^{2}}}, \\
\omega_{2 \pm}=\kappa U \pm \sqrt{\frac{A+\sqrt{A^{2}-4 B}}{2 \delta^{2}}}, \\
\omega_{3}=\kappa U
\end{gathered}
$$

where

$$
A=\frac{\delta^{2} \kappa^{2}}{1-\alpha_{0}}+\omega_{B}^{2}+\frac{3 \alpha_{0}}{1-\alpha_{0}}
$$



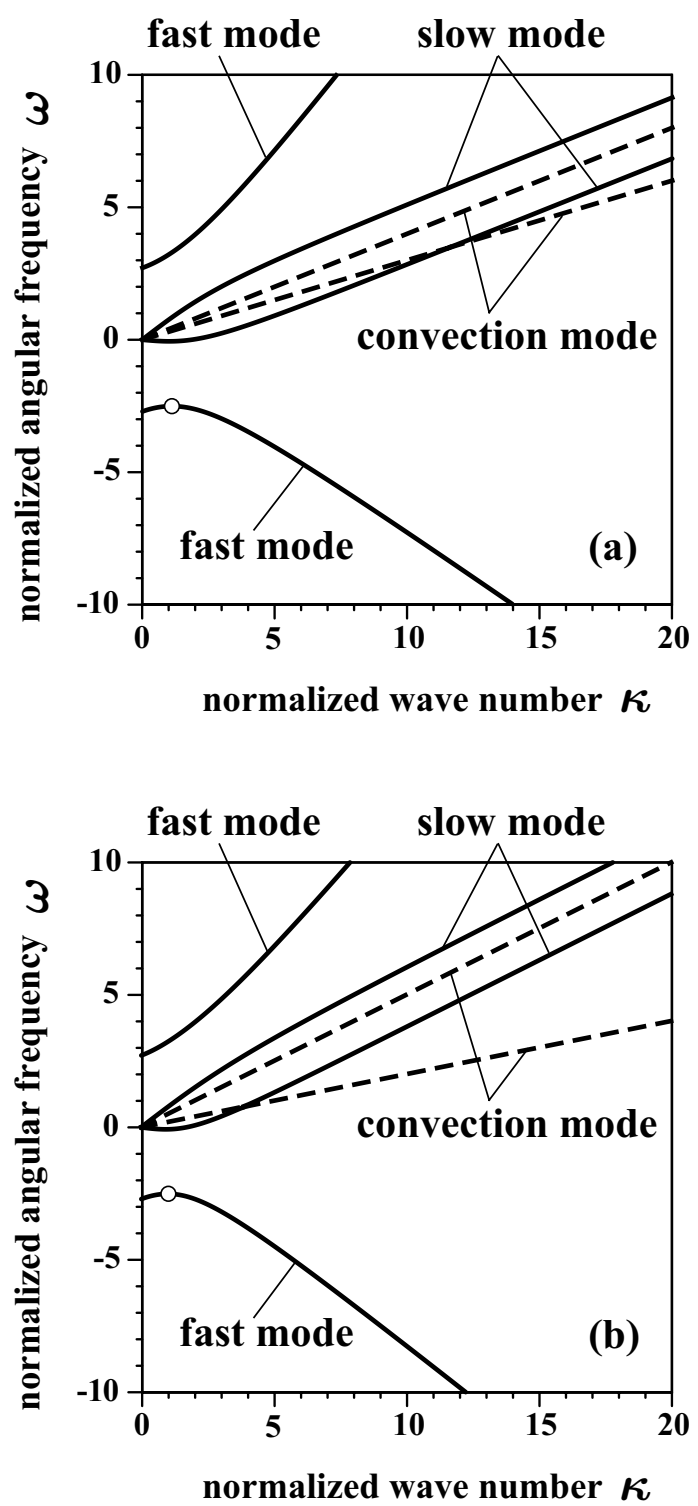

Fig. 2. The dispersion relations of the six propagation modes in the bubbly flows with different gasand liquid-phase velocities. The open circle indicates the maximum of the fast mode. (a): $\delta=0.01$, $\alpha_{0}=0.0002, u_{G 0}=0.4, u_{L 0}=0.3 ;(\mathrm{b}): \delta=0.01, \alpha_{0}=0.0002, u_{G 0}=0.5, u_{L 0}=0.2$. The virtual mass coefficients are set as $\beta_{1}=\beta_{2}=\beta_{3}=\frac{1}{2}$ and $R_{0}=0.1 \mathrm{~mm}$ in both cases.

$$
B=\left[\omega_{B}^{2}+\frac{3 \alpha_{0}\left(3-2 \alpha_{0}\right) p_{G 0}}{1-\alpha_{0}}\right] \frac{\delta^{2} \kappa^{2}}{1-\alpha_{0}} .
$$

When $U=0$, eqs. (25) and (26) reduce to the results in the bubbly liquid uniform and at rest studied in the previous paper (Egashira et al. 2004), where $\omega_{1 \pm}$ and $\omega_{2 \pm}$ are, respectively, referred to as the slow mode and the fast mode. The third mode $\omega_{3}=\kappa U$ may be called a convection mode. The fact that the five $\omega$ 's are real for an arbitrary real $\kappa$ means that the one-dimensional steady flow of $u_{G 0}=u_{L 0}=U$ described by the present model is linearly stable 
J. Phys. Soc. Jpn.

FULL PAPER

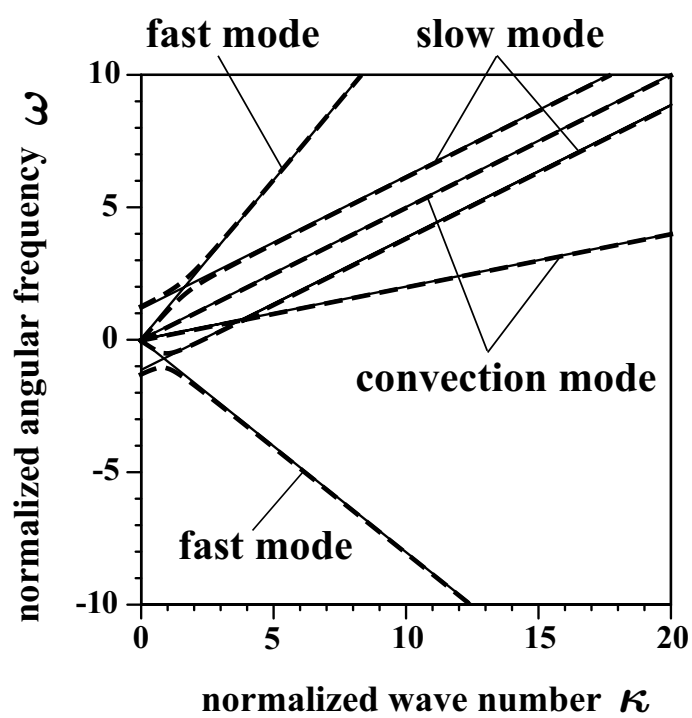

Fig. 3. The dispersion relation in the limit of $\alpha_{0} \rightarrow 0: u_{G 0}=0.5, u_{L 0}=0.2, \delta=0.01, \beta_{1}=\beta_{2}$, and $R_{0}=0.1 \mathrm{~mm}$. The dashed curves are those for $\alpha_{0}=0.00001$.

under the one-dimensional perturbations.

Figure 1 shows the dispersion relation of the fast and slow modes for $U=0$ studied by Egashira et al. (2004). The corresponding curves for $U \neq 0$ can be given by adding $\kappa U$.

\subsection{Waves in non-uniform flow}

In the case that $u_{G 0} \neq u_{L 0}$, the angular frequency $\omega$ cannot be expressed explicitly in terms of simple functions of $\kappa$. However, the stability of convection mode when the difference between $u_{G 0}$ and $u_{L 0}$ is sufficiently small can readily be examined in the following way.

Expanding $f(\kappa, \omega)$ around $\omega=\kappa U$ and $u_{G 0}=u_{L 0}=U$ with respect to $\omega, u_{G 0}$, and $u_{L 0}$ gives

$$
\begin{aligned}
f(\kappa, \omega)=\left.\frac{1}{2}(\omega-\kappa U)^{2} \frac{\partial^{2} f}{\partial \omega^{2}}\right|_{0}+ & \left.\frac{1}{2}\left(u_{G 0}-U\right)^{2} \frac{\partial^{2} f}{\partial u_{G 0}^{2}}\right|_{0} \\
+\left.\frac{1}{2}\left(u_{L 0}-U\right)^{2} \frac{\partial^{2} f}{\partial u_{L 0}^{2}}\right|_{0}+\left.(\omega-\kappa U)\left(u_{G 0}-U\right) \frac{\partial^{2} f}{\partial \omega \partial u_{G 0}}\right|_{0} & \\
+\left.(\omega-\kappa U)\left(u_{L 0}-U\right) \frac{\partial^{2} f}{\partial \omega \partial u_{L 0}}\right|_{0} & \\
& +\left.\left(u_{G 0}-U\right)\left(u_{L 0}-U\right) \frac{\partial^{2} f}{\partial u_{G 0} \partial u_{L 0}}\right|_{0}+O(3),
\end{aligned}
$$

where the partial derivative with the subscript 0 denotes the value evaluated at $\omega=\kappa U$ and $u_{G 0}=u_{L 0}=U$, and we have used the fact that

$$
\left.f(\kappa, \kappa U)\right|_{u_{G 0}=u_{L 0}=U}=\left.\frac{\partial f}{\partial \omega}\right|_{0}=\left.\frac{\partial f}{\partial u_{G 0}}\right|_{0}=\left.\frac{\partial f}{\partial u_{L 0}}\right|_{0}=0 .
$$


Without loss of generality, we can put $u_{G 0} \neq u_{L 0}=U$, and then we have

$$
\begin{aligned}
\left.f(\kappa, \omega) \cong \frac{1}{2}(\omega-\kappa U)^{2} \frac{\partial^{2} f}{\partial \omega^{2}}\right|_{0}+\left.\frac{1}{2}\left(u_{G 0}-U\right)^{2} \frac{\partial^{2} f}{\partial u_{G 0}^{2}}\right|_{0} & \\
& +\left.(\omega-\kappa U)\left(u_{G 0}-U\right) \frac{\partial^{2} f}{\partial \omega \partial u_{G 0}}\right|_{0}=0 .
\end{aligned}
$$

Clearly, eq. (32), the quadratic equation for $\omega$, has two real roots, if and only if an inequality

$$
\left(\left.\frac{\partial^{2} f}{\partial \omega \partial u_{G 0}}\right|_{0}\right)^{2} \geqq\left(\left.\frac{\partial^{2} f}{\partial \omega^{2}}\right|_{0}\right)\left(\left.\frac{\partial^{2} f}{\partial u_{G 0}^{2}}\right|_{0}\right),
$$

holds. The partial derivatives in eq. (33) can be given from the complete form of $f(\kappa, \omega)$ as

$$
\begin{gathered}
\left.\frac{\partial^{2} f}{\partial \omega^{2}}\right|_{0}=\frac{2 \kappa^{2} p_{L 0}}{\delta^{2}}\left[\beta_{1}\left(\omega_{B}^{2}+2 \alpha_{0} \hat{\sigma}\right)+\left(\alpha_{0}-\alpha_{0}^{2}\right)\left(\omega_{B}^{2}+2 \hat{\sigma}\right)\right], \\
\left.\frac{\partial^{2} f}{\partial u_{G 0}^{2}}\right|_{0}=\frac{2 \kappa^{4} p_{L 0}}{\delta^{2}}\left(1-\alpha_{0}\right)\left(\beta_{1}-\beta_{2}\right)\left(\omega_{B}^{2}+2 \alpha_{0} \hat{\sigma}\right), \\
\left.\frac{\partial^{2} f}{\partial \omega \partial u_{G 0}}\right|_{0}=\frac{\kappa^{3} p_{L 0}}{\delta^{2}}\left(\alpha_{0}-1\right)\left(2 \beta_{1}-\beta_{2}\right)\left(\omega_{B}^{2}+2 \alpha_{0} \hat{\sigma}\right) .
\end{gathered}
$$

Note that the above three derivatives are independent of $U$ and $\beta_{3}$.

In the simplest case $\beta_{1}=\beta_{2}$, the inequality (33) holds for arbitrary real $\kappa$ and $U$, and the solution of (32) for $\beta_{1}=\beta_{2}$ is given as

$$
\omega=\left\{\begin{array}{l}
\kappa U, \\
\kappa\left[U-\frac{C}{D}\left(u_{G 0}-u_{L 0}\right)\right],
\end{array}\right.
$$

where

$$
\begin{gathered}
C=\beta_{1}\left(1-\alpha_{0}\right)\left(\omega_{B}^{2}+2 \alpha_{0} \hat{\sigma}\right), \\
D=\beta_{1} \omega_{B}^{2}+\alpha_{0}\left[\omega_{B}^{2}+2\left(1+\beta_{1}\right) \hat{\sigma}\right]-3 \alpha_{0}^{2}\left(p_{L 0}+2 \hat{\sigma}\right) .
\end{gathered}
$$

The same $\omega$ as eq. (37) with eqs. (38) and (39) can be obtained even if we put $u_{G 0}=U$ in eq. (30). As a result, it is found that the convection mode when the difference between $u_{G 0}$ and $u_{L 0}$ is sufficiently small is the stable propagation mode for arbitrary real $\kappa$, if the inequality (33) holds. In other words, the bubbly flows with different gas- and liquid-phase velocities are stable if the difference in flow velocities are sufficiently small. The third coefficients of virtual mass force $\beta_{3}$ does not affect the above results.

Simple analytical approach as shown above cannot be applied to the case that the difference in the flow velocities is not small. It is, however, easy to examine the dispersion relation numerically. The dispersion relations for the six propagation modes are numerically evaluated and plotted in Fig. 2. As shown in the figure, all the six modes seem to be stable for moderately small $\alpha_{0}$ in the entire range of $\kappa$, even if the difference in the flow velocities is not very small. Further numerical examination suggests that the angular frequency of convection mode 
does not become complex in the wavenumber range shown in eq. (23).

Incidentally, one can see two important features in Fig. 2: one is the intersection of the slow mode and convection mode, which suggests the existence of interaction of these two modes in the nonlinear problem. The other is the maximum in the $\omega-\kappa$ curve of fast mode propagating in the opposite direction to the bubbly flow, denoted by an open circle. Since the maximum means the zero group velocity, this may lead to a kind of localization of the wave energy of corresponding wavenumber component, and cause some interesting nonlinear phenomenon. The nonlinear problem will be studied in a future work.

\subsection{The limit of infinitesimal void fraction}

In the limit of $\alpha_{0} \rightarrow 0$, the linearized mass and momentum conservation equations for liquid phase, eqs. (14) and (16), are simplified as

$$
\begin{gathered}
\frac{\partial}{\partial t}\left(p_{L 0} p_{L}\right)+\frac{\partial}{\partial x}\left(p_{L 0} u_{L 0} p_{L}+u_{L}\right)=0, \\
\frac{\partial u_{L}}{\partial t}+\frac{\partial}{\partial x}\left(p_{L 0} p_{L}+u_{L 0} u_{L}\right)=0
\end{gathered}
$$

which are the same as those for an inviscid compressible fluid with the Tait equation of state (8). The dispersion relation for the system (13), (40), (15), (41), (17), and (18) can then be given as

$$
\begin{aligned}
\left(\omega-\kappa u_{G 0}\right)\left[\delta^{2}\left(\omega-\kappa u_{G 0}\right)^{2}-\omega_{B}^{2}\right]\left[\left(\omega-\kappa u_{L 0}\right)^{2}\right. & \left.-\kappa^{2}\right] \\
& \times\left[\beta_{2} \kappa\left(u_{G 0}-u_{L 0}\right)+\beta_{1}\left(\omega-\kappa u_{G 0}\right)\right]=0 .
\end{aligned}
$$

Again, $\beta_{3}$ disappears in the dispersion relation. From eq. (42), we immediately have

$$
\begin{gathered}
\omega_{1 \pm}=\kappa u_{G 0} \pm \frac{\omega_{B}}{\delta}, \\
\omega_{2 \pm}=\kappa u_{L 0} \pm \kappa, \\
\omega_{3 \pm}=\left\{\begin{array}{l}
\kappa u_{G 0} \\
\kappa\left[u_{G 0}-\frac{\beta_{2}}{\beta_{1}}\left(u_{G 0}-u_{L 0}\right)\right],
\end{array}\right.
\end{gathered}
$$

where the slow mode $\omega_{1 \pm}$ represents the free oscillation of bubbles convected with $u_{G 0}$ and the fast mode $\omega_{2 \pm}$ is the acoustic wave in the liquid moving with $u_{L 0}$. The dispersion relation for each mode is shown in Fig. 3. As can be seen, the three modes in the limit $\alpha_{0} \rightarrow 0$ are almost equal to those for $\alpha_{0}=10^{-5}$. The fast and convection modes are almost dispersionless at $\alpha_{0}=10^{-5}$.

The expressions of the system of equations and the set of angular frequencies are now quite simple, and therefore we can explicitly write down the linear dispersive waves in the form suitable for the initial-value problem, 


$$
\begin{aligned}
q(x, t)=\sum_{n=1}^{3} \int_{-\infty}^{\infty} a_{n}(\kappa) \hat{q}_{n+}(\kappa) \exp \left[i \left(\omega_{n+} t\right.\right. & -\kappa x)] \mathrm{d} \kappa \\
& +\sum_{n=1}^{3} \int_{-\infty}^{\infty} b_{n}(\kappa) \hat{q}_{n-}(\kappa) \exp \left[i\left(\omega_{n-} t-\kappa x\right)\right] \mathrm{d} \kappa,
\end{aligned}
$$

where $a_{n}(\kappa)$ and $b_{n}(\kappa)(n=1,2,3)$ are the expansion coefficients, $q(x, t)$ is a column vector

$$
q(x, t)=\left(\begin{array}{c}
\alpha(x, t) \\
R(x, t) \\
p_{L}(x, t) \\
u_{G}(x, t) \\
u_{L}(x, t) \\
S(x, t)
\end{array}\right),
$$

and $\hat{q}_{n \pm}(\kappa)(n=1,2,3)$ are expressed as follows:

$$
\hat{q}_{1 \pm}(\kappa)=\left(\begin{array}{c}
3 i \delta\left[\mp 2 \beta_{1} \omega_{B}^{2} \pm \delta^{2} \kappa^{2} Q\right] \\
2 i \delta \omega_{B}\left(\mp \beta_{1} \omega_{B}-\beta_{2} \delta \kappa V\right) \\
0 \\
3 i \delta \omega_{B}\left[\delta \kappa Q \pm 2 \beta_{2} \omega_{B} V\right] \\
0 \\
2 \omega_{B}^{2}\left(\beta_{1} \omega_{B} \pm \beta_{2} \delta \kappa V\right)
\end{array}\right),
$$


J. Phys. Soc. Jpn.

$$
\left.\begin{array}{l}
V=u_{G 0}-u_{L 0}, \\
Q=\omega_{B}^{2}-p_{L 0}\left(=2 p_{L 0}+4 \hat{\sigma}\right), \\
T_{\mp}=\delta^{2} \kappa^{2}(V \mp 1)^{2}-\omega_{B}^{2}, \\
Y_{\mp}=\mp \beta_{1}+\left(\beta_{1}-\beta_{2}\right) V, \\
Z_{\mp}=-3 Q+2 T_{\mp}\left[\beta_{1}+\beta_{3}(V \mp 1) V\right] .
\end{array}\right\}
$$

The vectors $\hat{q}_{n \pm}(\kappa)$ may be regarded as eigenvectors associated with the eigenvalues $\omega_{n \pm}(\kappa)$.

From eqs. (48)-(50), one can see that the slow mode (48), which corresponds to the classical pressure wave in bubbly flows, is independent of the liquid-phase variables $p_{L}$ and $u_{L}$, whereas the fast mode (49) affects all the variables in gas and liquid phases. The convection mode (50) consists of only $\alpha$ and $u_{G}$ components, and hence it may deserve to be called the void wave.

Once an initial condition $q(x, 0)$ is given, we have from eq. (46)

$$
\begin{aligned}
\sum_{n=1}^{3}\left[a_{n}(\kappa) \hat{q}_{n+}(\kappa)+b_{n}(\kappa) \hat{q}_{n-}(\kappa)\right] & \\
& =\frac{1}{2 \pi} \int_{-\infty}^{\infty} q(x, 0) \mathrm{e}^{i \kappa x} \mathrm{~d} x .
\end{aligned}
$$

For the demonstration, we shall consider two simple examples: (i) $p_{L}(x, 0) \neq 0$ and the other initial values are all zero; (ii) $\alpha(x, 0) \neq 0$ and the other initial values are all zero. Furthermore, we assume $\beta_{1}=\beta_{2}=\beta_{3}$.

In the first example, the expansion coefficients are given as

$$
\begin{gathered}
a_{1}(\kappa)=\frac{i p_{L 0} \hat{p}_{L}(\kappa)}{\delta \omega_{B}^{2}\left[\left(\delta \kappa V+\omega_{B}\right)^{2}-\delta^{2} \kappa^{2}\right]}, \\
b_{1}(\kappa)=\frac{i p_{L 0} \hat{p}_{L}(\kappa)}{\delta \omega_{B}^{2}\left[\delta^{2} \kappa^{2}-\left(\delta \kappa V-\omega_{B}\right)^{2}\right]}, \\
a_{2}(\kappa)=\frac{i \hat{p}_{L}(\kappa)}{(1-V)\left[\omega_{B}^{2}-\delta^{2} \kappa^{2}(1-V)^{2}\right]}, \\
b_{2}(\kappa)=\frac{i \hat{p}_{L}(\kappa)}{(1+V)\left[\omega_{B}^{2}-\delta^{2} \kappa^{2}(1+V)^{2}\right]}, \\
a_{3}(\kappa)=\frac{2 p_{L 0}\left[3\left(\omega_{B}^{2}-p_{L 0}\right)+2 \beta_{1} \omega_{B}^{2} V^{2}\right] \hat{p}_{L}(\kappa)}{\omega_{B}^{2}\left(V^{2}-1\right)}, \\
b_{3}(\kappa)=-4 p_{L 0} \hat{p}_{L}(\kappa),
\end{gathered}
$$

where $\hat{p}_{L}(\kappa)$ is the Fourier transform of $p_{L}(x, 0)$. The second example immediately gives

$$
\begin{gathered}
a_{1}(\kappa)=a_{2}(\kappa)=b_{1}(\kappa)=b_{2}(\kappa)=b_{3}(\kappa)=0, \\
a_{3}(\kappa)=\hat{\alpha}(\kappa),
\end{gathered}
$$

where $\hat{\alpha}(\kappa)$ is the Fourier transform of $\alpha(x, 0)$. 


\subsection{Numerical examples}

In this subsection, we shall present typical examples obtained by solving the system of the linearized equations (13)-(18) for some initial conditions with a finite difference method. Figures 4 4 show the evolution of wave profiles of pressure, bubble radius, and void fraction. In these figures, wave profiles are plotted from $t=0$ to $t=50$, and the bold dashed line indicates the locus of wave front. The wave amplitudes in Figs. 4-7 are nondimensional and scaled by the same nondimensional factor.

The case that only the pressure perturbation is imposed at $t=0$ is shown in Figs. 4 and 5. The pressure profile in Fig. 4(a) is the fast mode, and the amplitudes of the waves of the bubble radius and void fraction are small in this case. Figure 5 is the case for $\alpha_{0}=10^{-5}$, and as shown in Fig. 3, the fast mode and convection mode are almost dispersionless, although the convection mode is not clearly seen.

Figures 6 and 7 are the case that the perturbation of void fraction only is applied at $t=0$. In the both figures, a convection mode appears and its amplitude is large compared with the other modes. In particular, only the void wave is strongly excited in the case of $\alpha_{0}=10^{-5}$ shown in Fig. 7. This corresponds to the analytical result, eqs. (59) and (60).

\section{Conclusions}

The detailed analysis of the linear dispersive wave in bubbly flows has been carried out based on the averaged equations composed of conservation laws and equation of motion of bubble wall. The result suggests that all the three types of waves may be stable in the wavenumber range where the present set of averaged equations is applicable. Gavrilyuk and Saurel ${ }^{8)}$ have also obtained a similar stability result in a different method of analysis. Their model equation contains an analogue of Rayleigh-Plesset equation for bubble dynamics. ${ }^{17)}$ The bubble dynamics may have some important effect on the wave motion in the bubbly flows. 

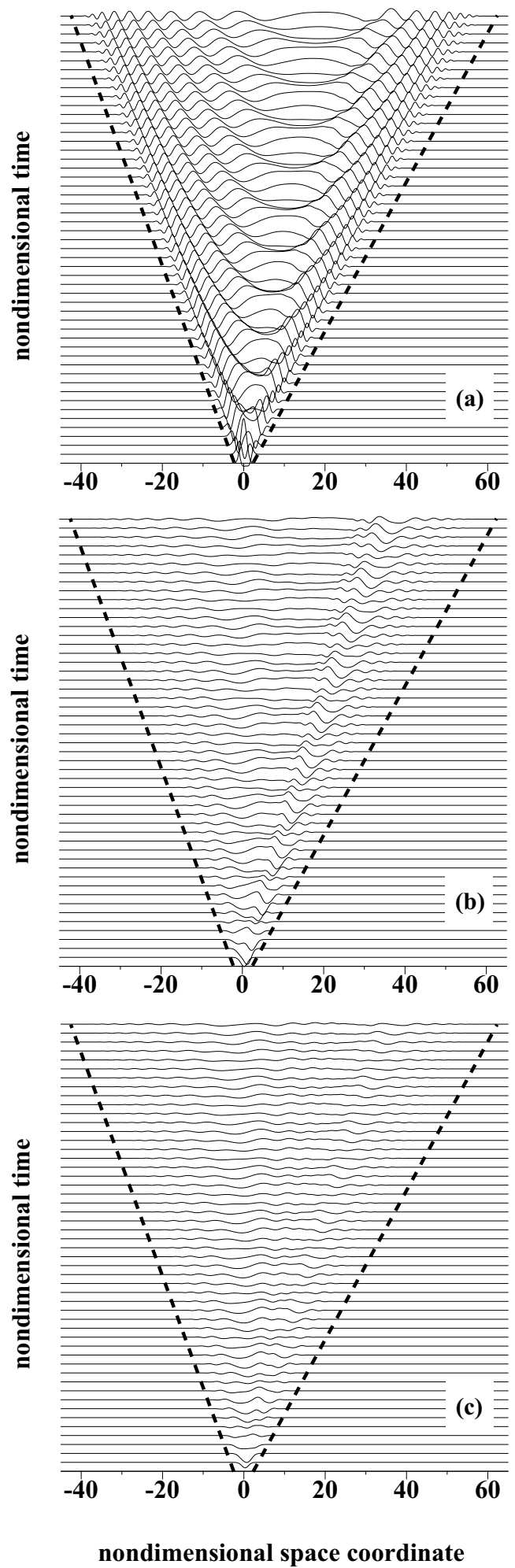

Fig. 4. Wave profiles in the case of $\alpha_{0}=0.0002, u_{G 0}=0.5, u_{L 0}=0.2, \delta=0.01, \beta_{1}=\beta_{2}=\beta_{3}=0.5$, and $R_{0}=0.1 \mathrm{~mm}$ : (a) pressure, (b) bubble radius, (c) void fraction. The initial condition is $p_{L}=\exp \left(-x^{2}\right)$ and $\alpha=R=u_{L}=u_{G}=S=0$ at $t=0$. 

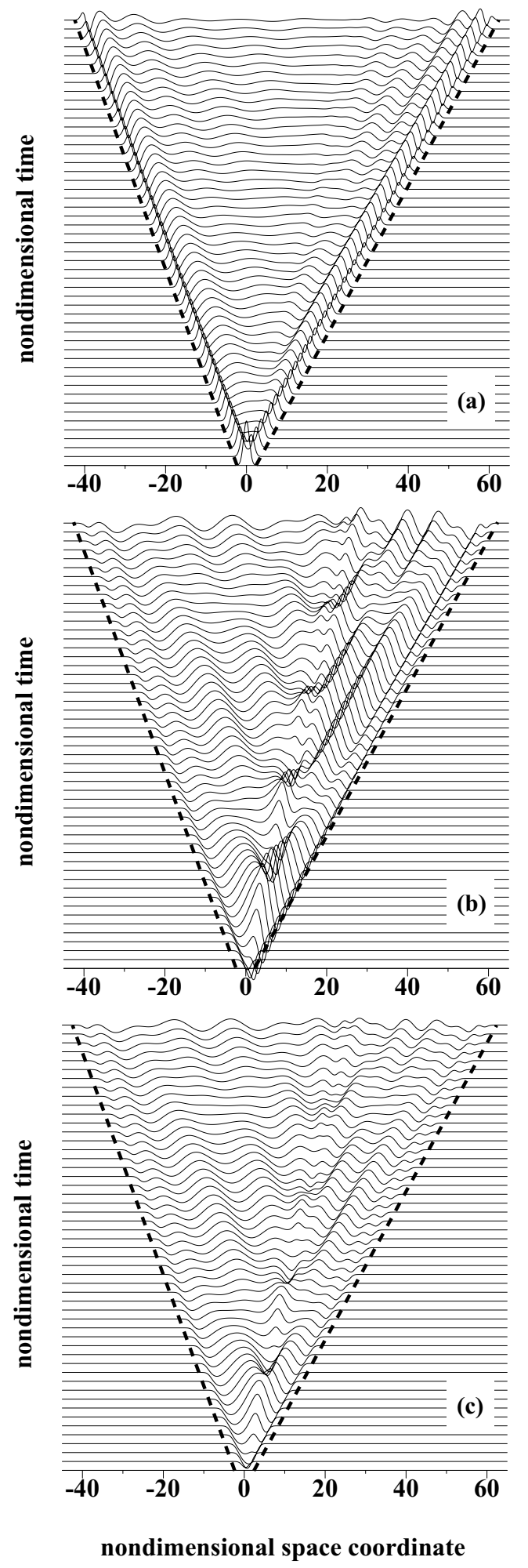

Fig. 5. Wave profiles in the case of $\alpha_{0}=0.00001, u_{G 0}=0.5, u_{L 0}=0.2, \delta=0.01, \beta_{1}=\beta_{2}=\beta_{3}=0.5$, and $R_{0}=0.1 \mathrm{~mm}$ : (a) pressure, (b) bubble radius, (c) void fraction. The initial condition is $p_{L}=\exp \left(-x^{2}\right)$ and $\alpha=R=u_{L}=u_{G}=S=0$ at $t=0$. 


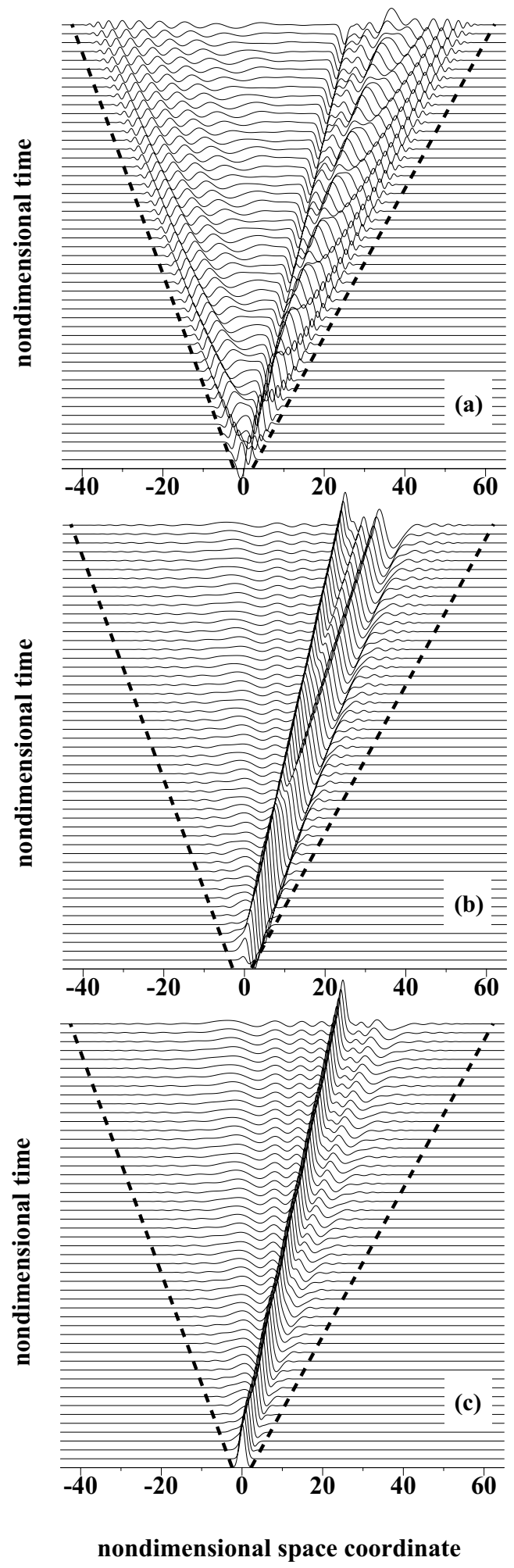

Fig. 6. Wave profiles in the case of $\alpha_{0}=0.0002, u_{G 0}=0.5, u_{L 0}=0.2, \delta=0.01, \beta_{1}=\beta_{2}=\beta_{3}=0.5$, and $R_{0}=0.1 \mathrm{~mm}$ : (a) pressure, (b) bubble radius, (c) void fraction. The initial condition is $\alpha=\exp \left(-x^{2}\right)$ and $R=p_{L}=u_{L}=u_{G}=S=0$ at $t=0$. 

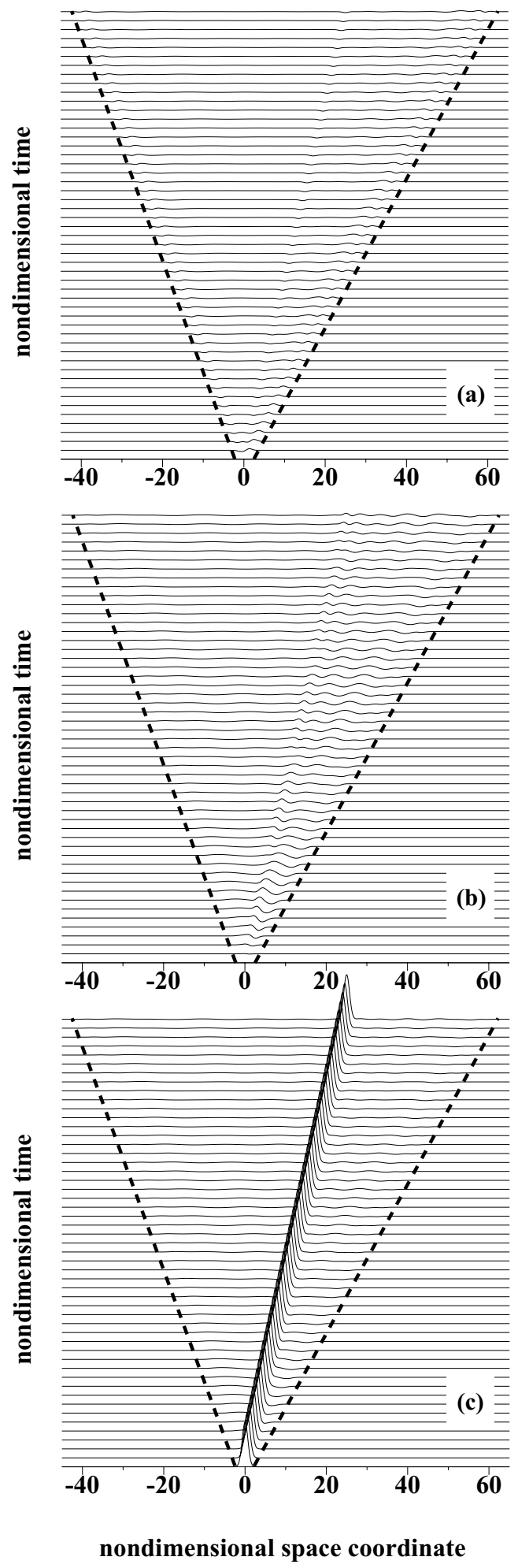

Fig. 7. Wave profiles in the case of $\alpha_{0}=0.00001, u_{G 0}=0.5, u_{L 0}=0.2, \delta=0.01, \beta_{1}=\beta_{2}=\beta_{3}=0.5$, and $R_{0}=0.1 \mathrm{~mm}$ : (a) pressure, (b) bubble radius, (c) void fraction. The initial condition is $\alpha=\exp \left(-x^{2}\right)$ and $R=p_{L}=u_{L}=u_{G}=S=0$ at $t=0$. 


\section{References}

1) L. van Wijngaarden: J. Fluid Mech. 33 (1968) 465.

2) R. E. Caflisch, M. J. Miksis, G. C. Papanicolaou and L. Ting: J. Fluid Mech. 153 (1985) 259.

3) C. Pauchon and S. Banerjee: Int. J. Multiphase Flow 12 (1986) 559.

4) C. Pauchon and S. Banerjee: Int. J. Multiphase Flow 14 (1988) 253.

5) K. W. Commander and A. Prosperetti: J. Acoust. Soc. Am. 85 (1989) 732.

6) R. T. Lahey, Jr.: AIChE J. 37 (1991) 123.

7) J.-W. Park, D. A. Drew and R. T. Lahey, Jr.: Int. J. Multiphase Flow 24 (1998) 1205.

8) S. Gavrilyuk and R. Saurel: J. Comp. Phys. 175 (2002) 326.

9) R. Egashira, T. Yano and S. Fujikawa: Fluid Dyn. Res. 34 (2004) 317.

10) J. B. Keller and I. I. Kolodner: J. Appl. Phys. 27 (1956) 1152.

11) A. V. Jones and A. Prosperetti: Int. J. Multiphase Flow 11 (1985) 133.

12) D. A. Drew and S. L. Passman: Theory of Multicomponent Fluids, (Springer, New York, 1999).

13) M. Kameda and Y. Matsumoto: Phys. Fluids 8 (1996) 322.

14) D. Z. Zhang and A. Prosperetti: J. Fluid Mech. 267 (1994) 185.

15) I. Eames and J. C. R. Hunt: J. Fluid Mech. 505 (2004) 349.

16) D. Drew, L. Cheng and R. T. Lahey, Jr.: Int. J. Multiphase Flow 5 (1979) 233.

17) M. S. Plesset and A. Prosperetti: Ann. Rev. Fluid Mech 9 (1977) 145. 